Algebraic $8 \mathcal{G}$ Geometric $\mathcal{T}$ opology

Volume 4 (2004) 1041-1044

Published: 3 November 2004

ATG

\title{
Mp-small summands increase knot width
}

\author{
JACOB HENDRICKS
}

\begin{abstract}
Scharlemann and Schultens have shown that for any pair of knots $K_{1}$ and $K_{2}, w\left(K_{1} \# K_{2}\right) \geq \max \left\{w\left(K_{1}\right), w\left(K_{2}\right)\right\}$. Scharlemann and Thompson have given a scheme for possible examples where equality holds. Using results of Scharlemann-Schultens, Rieck-Sedgwick and Thompson, it is shown that for $K=\#_{i=1}^{n} K_{i}$ a connected sum of mp-small knots and $K^{\prime}$ any non-trivial knot, $w\left(K \# K^{\prime}\right)>w(K)$.
\end{abstract}

AMS Classification 57M25; 57M27

Keywords Thin position, knot width

\section{Introductory remarks}

Thin position, introduced by David Gabai [1], has applications in solving difficult problems, yet there remains much to understand about the width of knots. Current understanding of the behavior of width under connect sum is incomplete, but has some form. For example, given two knots $K_{1}$ and $K_{2}$, it is easily seen that $w\left(K_{1} \# K_{2}\right) \leq w\left(K_{1}\right)+w\left(K_{2}\right)-2$; simply stack the knots in thin position, perform the connect sum, and calculate the upperbound. Also, Rieck and Sedgwick [3] have shown that if $K_{1}$ and $K_{2}$ are mp-small (see definition 2.10 then $w\left(K_{1} \# K_{2}\right)=w\left(K_{1}\right)+w\left(K_{2}\right)-2$. By means of a fascinating method (see [4]), it has also been shown that for any two knots $K_{1}$ and $K_{2}$, $w\left(K_{1} \# K_{2}\right) \geq \max \left\{w\left(K_{1}\right), w\left(K_{2}\right)\right\}$. It is natural to wonder if there exists a pair of knots such that $w\left(K_{1} \# K_{2}\right)=w\left(K_{1}\right)$, for this would be a peculiar property of width. In fact, a class of possible examples of equality is presented in [5], where it is argued, though not proven, that for any knot $K_{2}$ there is a (typically quite complicated) knot $K_{1}$, apparently determined by the bridge number of $K_{2}$, so that $w\left(K_{1} \# K_{2}\right)=w\left(K_{1}\right)$. In contrast, we show here that if $K_{1}$ is the connect sum of mp-small knots, then for any knot $K_{2}, w\left(K_{1} \# K_{2}\right)>w\left(K_{1}\right)$.

I would like to thank the referees for many helpful remarks and Yo'av Rieck for many helpful conversations. 


\section{Preliminaries}

For the sake of brevity, familiarity with width, thin/thick levels, thin position, bridge position, swallow-follow torus, satellite knot as well as the following definitions and theorems will be assumed. One can find definitions of the italicized words in many sources, for example [2] and 4].

Definition 2.1 A knot $K \subset S^{3}$ is called meridionally planar small (mp-small) if the only incompressible meridional surface in its complement is a boundaryparallel annulus.

In [6], Thompson proved:

Theorem 2.1 (Thompson) If a knot $K$ in thin position countains a thin level, then it is not mp-small.

Thus, for an mp-small knot $K$, thin position must equal bridge position. In $\underline{3}$. (Theorem 4.1), a converse of Theorem 2.1 is given in the case that $K$ is the connect sum of two non-trivial knots.

Theorem 2.2 (Rieck-Sedgwick) Let $K$ be a connected sum of non-trivial knots, $K=K_{1} \# K_{2}$. Then any thin position for $K$ is not bridge position for $K$.

The following was also shown in [3]:

Theorem 2.3 (Rieck-Sedgwick) Let $K=\#_{i=1}^{n} K_{i}$ be a connected sum of mp-small knots. If $K$ is in thin position, then there is an ordering of the summands $K_{i_{1}}, K_{i_{2}}, \ldots, K_{i_{n}}$ and a collection of leveled decomposing annuli $A_{i_{1}}, A_{i_{2}}, \ldots, A_{i_{n}-1}$ so that the thin levels of the presentation are precisely the annuli $\left\{A_{i_{j}}\right\}$ occurring in order, where the annulus $A_{i_{j}}$ separates the connected sum $K_{i_{1}} \# K_{i_{2}} \# \ldots \# K_{i_{j}}$ from the connected sum $K_{i_{j}+1} \# \ldots \# K_{i_{n}}$.

Scharlemann-Schultens 4] describe a method for reimbedding a knot $K$ so that a height function on $K$ would be preserved.

Theorem 2.4 (Scharlemann-Schultens) Suppose $p: S^{3} \rightarrow \mathbb{R}$ is the standard height function. Let $K \subset S^{3}$ be a knot that lies inside a standard unknotted torus $H \subset S^{3}$. Let $f: H \rightarrow S^{3}$ be a possibly knotted embedding of $H$ in $S^{3}$. Then there is a reimbedding $f^{\prime}: H \rightarrow S^{3}$ so that 
(1) $p f^{\prime}=p f$, i.e. the reimbedding preserves height

(2) $S^{3}-f^{\prime}(H)$ is a solid torus (so $f^{\prime}(H)$ is an unknotted solid torus) and

(3) $f\left(K^{\prime}\right)$ is isotopic to $K$ in $S^{3}$.

This is a specific case of what is shown in Corollary 5.4 of [4. In this paper will apply the theorem in this setting: We will be given a connected sum $K \# L$ embedded in $S^{3}$ via a knotted embedding $f: H \rightarrow S^{3}$. $K$ has here been placed in $H$ as a wrapping number one knot and the embedding $f$ is known to take the core of $H$ to the knot $L$. (The boundary of $f(H)$ is commonly called a "swallow-follow" torus for $K \# L$, for it swallows $K$ and follows $L$.)

\section{When summands are mp-small}

In [4, it is shown that $w\left(K_{1} \# K_{2}\right) \geq w\left(K_{i}\right)$ for $i=1,2$. One may ask if there is a pair of knots $K_{1}$ and $K_{2}$ such that equality holds (eg. [5]).

It is shown in [3] that for $K_{1}$ and $K_{2}$ both mp-small knots, $w\left(K_{1} \# K_{2}\right)=$ $w\left(K_{1}\right)+w\left(K_{2}\right)-2$. In particular, when both are non-trivial knots, $w\left(K_{1} \# K_{2}\right)$ $>w\left(K_{i}\right), i=1,2$. A natural question is whether this inequality remains true if only one of the knots is mp-small.

Proposition 3.1 If $K_{1}$ is an mp-small knot, then for any non-trivial knot $K_{2}$, $w\left(K_{1} \# K_{2}\right)>w\left(K_{1}\right)$.

Proof Since $K_{1}$ is mp-small, Theorem 2.1 implies that thin position of $K_{1}$ equals bridge position. Then, take $K_{1} \# K_{2}$ to be a satellite knot with companion knot $K_{2}$ via the swallow-follow torus. Put $K_{1} \# K_{2}$ in thin position; notice by Theorem 2.2. $K_{1} \# K_{2}$ cannot be in bridge position, so there exists a thin level. By Theorem 2.4 there exists a height preserving reimbedding that changes the knot $K_{1} \# K_{2}$ to the knot $K_{1}$. Since $K_{1} \# K_{2}$ has a thin level, so must a height preserving reimbedding of $K_{1} \# K_{2}$. So, this reimbedding yields an embedding of $K_{1}$ that has a thin level. (i.e. an embedding of $K_{1}$ that is not in bridge position.) It follows that this embedding of $K_{1}$ cannot be in thin position, so its width as embedded, and hence the width of $K_{1} \# K_{2}$, is greater than the minimal width of $K_{1}$.

One can use the method for showing proposition 3.1 to prove a more general statement; that is, $K_{1}$ can be taken to be the connect sum of mp-small knots. 
Theorem 3.1 Let $K=\#_{i=1}^{n} K_{i}$ be a connected sum of mp-small knots and let $K^{\prime}$ be any non-trivial knot. Then, $w\left(K \# K^{\prime}\right)>w(K)$.

Proof Suppose $w\left(K \# K^{\prime}\right)=w(K)$; we will show that $K^{\prime}$ must be the unknot. Now, take $K^{\prime}$ to be the companion knot of the satellite knot given by $K \# K^{\prime}$ with $H$ a torus that swallows $K$ and follows $K^{\prime}$. Put $K \# K^{\prime}$ in thin position. By Theorem 2.4 there exists a reimbedding $f$ such that $f$ preserves a height function on $K \# K^{\prime}$ and $f\left(K \# K^{\prime}\right)$ is isotopic to $K$ in $S^{3}$. We are assuming $w\left(K \# K^{\prime}\right)=w(K)$, thus $f\left(K \# K^{\prime}\right)$ is a thin presentation of $K$; therefore, by Theorem [2.3. every thin level of $K$ is one of the $n-1$ decomposing annuli, which decompose the connect sum $\#_{i=1}^{n} K_{i}$ into mp-small knots. Since each $K_{i}$ is mp-small, by Theorem 2.1, thin position of each $K_{i}$ is bridge position of each $K_{i}$. Now, since $f$ is a height preserving reimbedding, $K \# K^{\prime}$ must have $n-1$ decomposing annuli that constitute all of the thin levels; hence, $K \# K^{\prime}=\#_{i=1}^{n} K_{i}^{\prime}$. Note that each of the $n$ components $K_{i}^{\prime}$ is in bridge position. This must also be thin position; for if not, one could thin $K \# K^{\prime}$ by thinning the summand for which bridge does not equal thin, but we have put $K \# K^{\prime}$ in thin position. It follows from Theorem 2.2 that each $K_{i}^{\prime}$ must be prime. Hence, we have $K \# K^{\prime}=\#_{i=1}^{n} K_{i}^{\prime}$ where each $K_{i}^{\prime}$ is prime, and $K=\#_{i=1}^{n} K_{i}$ where each $K_{i}$ is prime. Since knot factorizations are unique, $K^{\prime}$ must be the unknot.

\section{References}

[1] D Gabai, Foliations and the topology of 3-manifolds, III, J. Differential Geom. 26 (1987) 479-536 MathReview

[2] D J Heath, T Kobayashi, Essential Tangle Decomposition from Thin Position of a Link, Pacific J. Math. 179 (1997) 101-117 MathReview

[3] Y Rieck, E Sedgwick, Thin position for a connected sum of small knots, Algebr. Geom. Topol. 2 (2002) 297-309 MathReview

[4] M Scharlemann, J Schultens, 3-manifolds with planar presentations and the width of satellite knots, arXiv:math.GT/0304271

[5] M Scharlemann, A Thompson, On the additivity of knot width, from: "Proceedings of the Casson Fest", (Cameron Gordon and Yoav Rieck, editors), Geom. Topol. Monogr. 7 (2004) 135-144

[6] A Thompson, Thin position and bridge number for knots in the 3-sphere, Topology 36 (1997) 505-507 MathReview

Department of Mathematics, University of Arkansas, Fayetteville, AR 72701, USA

Email: jghendr@uark.edu Received: 14 June 2004 Revised: 20 August 2004 